\title{
Lattice Boltzmann modelling of liquid distribution in unsaturated granular media Vincent Richefeu ${ }^{a}$, Farhang Radjai ${ }^{\mathrm{b}, \mathrm{c}}$, Jean-Yves Delenne ${ }^{\mathrm{d}, *}$
}

\author{
a 3SR, UMR 5521 UJF-Grenoble 1, Grenoble-INP, CNRS UMR 5521, 38041 Grenoble Cedex 9, France \\ ${ }^{\mathrm{b}}$ LMGC, UMR 5508 CNRS-University Montpellier 2, 34095 Montpellier, France \\ ${ }^{c}<M S E>^{2}$, UMI 3466 CNRS-MIT, DCEE, Massachusetts Institute of Technology, 77 Massachusetts Avenue, Cambridge, CA 02139, USA
}

${ }^{\mathrm{d}}$ IATE, UMR 1208 INRA-CIRAD-Montpellier Supagro-University of Montpellier, 34060 Montpellier Cedex, France

\begin{abstract}
A B S T R A C T
We use capillary condensation simulated by a multiphase Lattice Boltzmann model as a means to gener-ate homogeneous distributions of liquid clusters in 2D granular media. Liquid droplets condense from the vapour phase between and on the grains, and they transform into capillary bonds and liquid clusters as thermodynamic equilibrium is approached. As the amount of condensed liquid is increased, liquid clus-ters of increasing connectivity are formed and the distribution of liquid undergoes topological transitions until the whole pore space is filled by the liquid. We investigate the cluster statistics and local grain envi-ronments. From extensive simulations, we also obtain the mean Laplace pressure as a function of the amount of liquid, which is found to be quite similar to the well-known experimental retention curve in soil mechanics. The tensile stress carried by the grains increases as a function of the amount of con-densed liquid up to a peak in the funicular state beyond which the stress falls off as a result of pressure drop inside the merging clusters.
\end{abstract}

Keywords:

Unsaturated soils

Granular materials

Capillary condensation

Lattice Boltzmann Method

\section{Introduction}

Granular media in nature and industry often occur as threephase materials composed of grains, liquid and air. For example, the triggering of landslides and sediment transport involve the rheology of dense mixtures of grains and water [1]. The slope stability by vegetation is also governed by the hydro-mechanical properties of the root-soil matrix [2]. Another example is the so-called vadoze zone (above the phreatic zone) where water is retained by adhesion and capillary action without being necessarily saturated. The properties of this zone are essential for agriculture and pollutant transport. In the same way, the hydro-textural evolution of wetted granular materials is an important problem in industrial processes such as wet mixing and agglomeration of ores and powders [3].

An outstanding effect of liquid is the bulk cohesion induced by surface tension in a partially wetted granular material. The stability of a sand castle, for example, is ensured by small amounts of water distributed in the form of menisci that hold the grains together at their contact points [4]. The shear strength increases

\footnotetext{
* Corresponding author.

E-mail addresses: Vincent.Richefeu@3sr-grenoble.fr (V. Richefeu), franck. radjai@univ-montp2.fr, fradjai@mit.edu (F. Radjai), delenne@supagro.inra.fr (J.-Y. Delenne).
}

rapidly in this pendular state until all contacts between neighbouring grains are wetted. Beyond this point, the cohesive strength of the material remains nearly independent of the amount of liquid. Further increase of liquid volume leads to liquid clustering and increasing number of grains fully immersed in the liquid phase. The distribution of liquid in this funicular state and the dependence of capillary cohesion on the amount of liquid are only beginning to be explored [5]. Recent experiments show that the pore-filling process in transition to the funicular state is a rich and interesting physics problem involving an intricate texturing of liquid clusters [6]. The intermediate states in transition to a fully saturated state have been characterised in soil mechanics through experimental retention curves, and the authors are aware of no systematic studies of shear strength and liquid distribution at the particle scale [7].

Numerical simulation of capillary liquid requires a multiphase approach and it has been applied in a recent paper to unsaturated granular materials by Delenne et al. using a Lattice Boltzmann model (LBM) in 2D [8]. There, we investigated the statistics of liquid clusters and the Laplace pressure as a function of the amount of liquid. In the present paper, we introduce the algorithmic aspects of the single-component multi-phase LBM and postanalysis methods in application to granular materials. The liquid is introduced by condensation from a vapour phase. This method yields a homogeneous distribution of liquid bridges between 
grains at thermodynamic equilibrium. By slowly increasing the amount of vapour, we obtain different states from pendular bridges to a fully saturated state. We show that the three parts of the retention curve are correctly reproduced by simulations and they reflect the evolution of the wet coordination number of grains and the connectivity of liquid clusters. We further discuss the cohesive stress induced by negative capillary pressures on the grains and its evolution with the amount of liquid.

\section{Multiphase LBM in a granular packing}

The simulation of partially saturated states in a granular material requires three ingredients: (1) Dynamics of solid grains generally simulated by a discrete element method (DEM) [9,10], (2) Navier-Stokes equations for liquid and gas [11], and (3) thermodynamics of gas-liquid phase transition [12]. The Navier-Stokes equations can be simulated through the LBM [13], which consists in discretising the Boltzmann equation on a regular lattice with an adequate collision operator. This is a computationally efficient method and, as we shall see below, it allows for direct incorporation of an equation of state.

We consider here a two-dimensional model defined on a square grid of spacing $\Delta x$ as shown in Fig. 1. In this model, eight directions of motion are defined between each node and its surrounding neighbours. This 2D lattice is endowed with nine discrete velocities, and for this reason it is called D2Q9. These velocities are expressed in terms of the so-called lattice speed $c=\Delta x / \Delta t$, where $\Delta t$ is the time step. For the velocities attributed to the eight lattice directions, we set $\vec{e}_{1}=(c, 0), \vec{e}_{2}=(0, c), \vec{e}_{3}=(-c, 0), \vec{e}_{4}=(0,-c)$ for the rectilinear directions, and $\vec{e}_{5}=(c, c), \vec{e}_{6}=(-c, c), \vec{e}_{7}=$ $(-c,-c), \vec{e}_{8}=(c,-c)$ for the diagonal directions. These are fluid flow velocities between neighbouring nodes. The fluid particles remaining at a node are given a zero velocity $\vec{e}_{0}=(0,0)$.

The fluid flow is governed by the discretised evolution equation:

$f_{a}\left(\vec{x}_{a}, t+\Delta t\right)=f_{a}(\vec{x}, t)+\Omega_{a}(\vec{x}, t)+\Delta f_{a}(\vec{x}, t)$

where the main variable $f_{a}$ is the fluid distribution function along the direction $a$ (with $a=0$ corresponding to the in-node distribution), and $\vec{x}_{a}=\vec{x}+\vec{e}_{a} \Delta t$ denotes the neighbouring nodes. The mass density at a node $\vec{x}$ is given by the sum $\rho(\vec{x}, t)=\sum_{a=0}^{8} f_{a}$. The collision operator $\Omega_{a}(\vec{x}, t)$ is based on the BGK approximation [14]:

$\Omega_{a}(\vec{x}, t)=\frac{\Delta t}{\tau}\left(f_{a}(\vec{x}, t)-\bar{f}_{a}(\vec{x}, t)\right)$

with a single relaxation time $\tau$. This approximation together with the discretized Lattice-Boltzmann Eq. (1) leads to Navier-Stokes equations [15].

The equilibrium densities $\bar{f}_{a}(\vec{x}, t)$ are given by the discretised Maxwell distribution

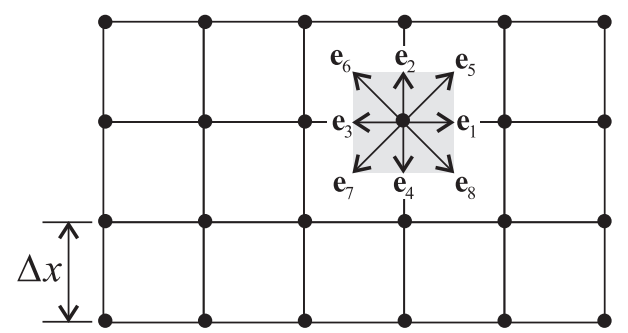

Fig. 1. Lattice Boltzmann discretization of space and D2Q9 model. $\bar{f}_{a}(\vec{x}, t)=w_{a} \rho\left[1+\frac{1}{c_{s}^{2}} \vec{e}_{a} \cdot \vec{u}(\vec{x}, t)+\frac{1}{2 c_{s}^{4}}\left[\vec{e}_{a} \cdot \vec{u}\right]^{2}(\vec{x}, t)-\frac{1}{2 c_{s}^{2}} \vec{u}^{2}(\vec{x}, t)\right]$

where the weight factor $w_{a}$ is $4 / 9$ for $a=0,1 / 9$ for $a=1, \ldots, 4$ and $1 / 36$ for $a=5 \ldots 8$ and $c_{s}=c / \sqrt{3}$ is the speed of sound. In Eq. (3), the fluid velocity $\vec{u}(\vec{x}, t)$ is given by

$\rho(\vec{x}, t) \vec{u}(\vec{x}, t)=\sum_{a=0}^{8} f_{a}(\vec{x}, t) \vec{e}_{a}+\vec{F}(\vec{x}, t) \Delta t / 2$

where $\vec{F}(\vec{x}, t)$ is a bulk force acting on the fluid at node $\vec{x}$ and at time $t$.

A major advantage of LBM is its ability to account for multiphase flows [16-18]. The interactions between liquid, gas and grains are derived using nonlocal potentials. These potentials control the surface tension and contact angle between fluid and solid [12]. Long-range potentials were initially introduced by Shan and Chen $[19,20]$ through a so-called effective mass $\psi$ reflecting the momentum change due to cohesion forces. In the early models of phase separation, $\psi$ was simply assumed to be an increasing function of density [21]. Recently, different methods have been proposed through a thermodynamically founded equation of state $[22,12,23]$. Most of these methods use the approximation

$\psi=\sqrt{2\left(\rho-p / c_{s}^{2}\right)}$

obtained through the Chapman-Enskog expansion, where $p$ is the pressure at a given node [24].

For the simulations presented in this paper, we used the following expression of the body force:

$\vec{F}(\vec{x})=\psi(\rho(\vec{x})) \sum_{a=0}^{8} w_{a}\left[\psi(\rho(\vec{x}))\left(1-s\left(\vec{x}_{a}\right)\right)+\psi\left(\rho_{s}(\vec{x})\right) s\left(\vec{x}_{a}\right)\right] \vec{e}_{a}$

where $s$ is an indicator function, equal to 1 if the node is solid and 0 otherwise, and $\rho_{s}(\vec{x})$ is a parameter controlling the contact angle at the fluid-solid interface [25]. This expression may account for both fluid internal cohesion and fluid-solid adhesion.

The thermodynamics of phase change is based on Carnahan-S tarling's equation of state [23]; see Fig. 2. It can be written using the variables $p, \rho$ and $T$, which are normalised here by their values at the critical point:

$p=\gamma \rho T \frac{1+\beta \rho+(\beta \rho)^{2}-(\beta \rho)^{3}}{(1-\beta \rho)}-\alpha \rho^{2}$

where $\alpha=3.8525, \beta=0.1304$ and $\gamma=2.7859$ are constant parameters obtained by solving the system of 3 equations: $\left\{p=1 ;\left.\frac{\partial p}{\partial \rho}\right|_{T}=0 ;\left.\frac{\partial^{2} p}{\partial^{2} \rho}\right|_{T}=0\right\}$ at critical point $(\rho=1 ; T=1)[23]$. In

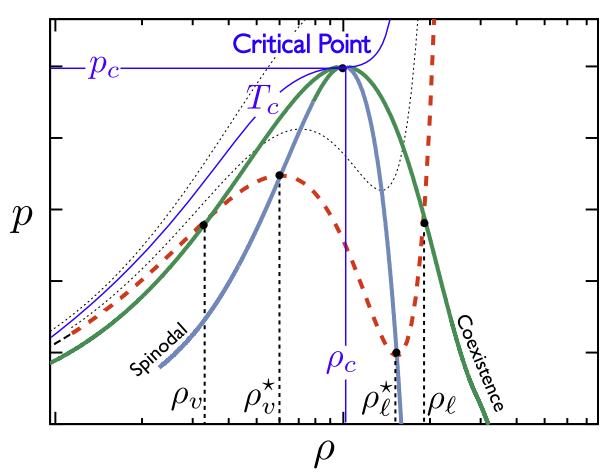

Fig. 2. Isotherm (dotted line), coexistence and spinodal curves according to Carnahan-Starling's equation of state. The pressures and densities are normalised by those of the triple point 
this model all free parameters are fixed by setting the values of the critical point. Moreover, for the computation, we use dimensionless Lattice Units (lu) by setting $\Delta t=1, \Delta x=1$.

\section{System description}

Without loss of generality, in our simulations we set the critical values to $p_{c}=0.00442 \mathrm{lu}, T_{c}=0.0943 \mathrm{lu}$ and $\rho_{c}=0.13044 \mathrm{lu}$ [24]. In the absence of grains, a uniform fluid of density equal to that of water vapour will condense into droplets if the temperature is set to a value below that of critical temperature and above the minimum spinodal density $\rho_{v}^{\text {; }}$; see Fig. 2 . But in order to trigger condensation, it is also necessary to add fluctuations in the fluid density field. Fig. 3 shows the condensation process in which smaller droplets coalesce rapidly into larger droplets. By varying the amount of vapour, we get drops of different sizes, from which we can determine the liquid-vapour surface tension $\gamma_{\ell v} \simeq 0.014 \mathrm{lu}$ from the Laplace equation, as shown in Fig. 4.

We used the same parameters for wetting of a granular material. The samples are prepared by isotropic compaction of 1000 disks with a friction coefficient $\mu=0.1$ simulated by the molecular dynamics method inside a biperiodic cell [10]. The grains have a uniform size distribution by volume fractions with a ratio 1.75 between the largest and smallest grain diameters. The resulting static configuration has a packing fraction 0.82 and a coordination number 5.6. The space is meshed by a rectilinear grid of 2,250,000 nodes, corresponding to at least 30 nodes within a small grain diameter. Furthermore, since in 2D the pores are closed, we added a single fluid node at the contact points between the particles to allow for liquid transport. This gap corresponds to a distance of the order of $\langle d\rangle / 40$. This is important for fast relaxation of thermodynamic variables to equilibrium although the vapour is introduced randomly in all pores. Note that all calculations are conducted by assuming that the grains are immobile. In practice, this implies that the confining pressure is high compared to capillary stress so that the condition of force balance on each grain remains unaffected as normal and tangential forces evolve.

In the simulations reported below, the wetting contact angle is set to zero. Physically, this is equivalent to setting $\gamma_{s v}=\gamma_{\ell v}$, where $\gamma_{s v}$ is the solid-vapour surface tension. This condition leads to condensation of liquid between grains. It can be shown that the fluid viscosity in LBM is given by $v=c_{s}^{2} \Delta t\left(\tau-\frac{1}{2}\right)$ [13]. We set $\tau=1$ so that in dimensionless units we have $v=1 / 6$. The corresponding value in physical units depends upon the time step and spatial resolution. For $d x=10^{-4} \mathrm{~m}$ and $d t=10^{-2} \mathrm{~s}$, the viscosity is that of water. Note that the stability of the calculations is assured by choosing a value of the lattice speed $c$ above the maximum fluid velocity in the simulations.

In order to initiate capillary condensation, the temperature is set to $0.7 T_{c}$. The injection rate is low enough to allow for a

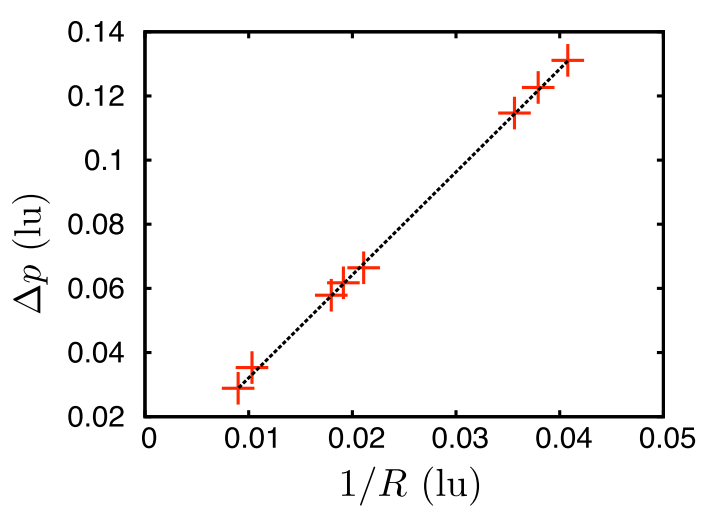

Fig. 4. Pressure drop across the liquid-gas interface as a function of the radius for 8 simulated drops and bubbles.

quasi-equilibrium state with a uniform injection of fluid at a constant rate in the pore space and continuous condensation of liquid between the particles. Fig. 5 shows the computed scalar fields of fluid density $\rho$, the potential $\psi$ and pressure $p$ in the granular material at thermodynamic equilibrium for $S \simeq 0.2$, where $S$ is defined as the volume $V_{\text {liquid }}$ of condensed liquid normalised by the total pore volume $V_{\text {pores }}$. We observe a net phase separation here in the pendular state with liquid bridges formed at all contact zones between grains. The fluid density $\rho$ at the interface between liquid and its vapour varies fast but smoothly over 4 lattice steps as shown in Fig. 6(a). A similar transition is observed at the solid-vapour interface shown in Fig. 6(b). Since we have $\gamma_{s v}=\gamma_{\ell v}$, the fluid density has the same value at the solid-liquid interface as in the liquid; see Fig. 6(c). Note that droplets and liquid layers of variable thickness can appear at the grain boundaries, but as the system tends to its thermodynamic equilibrium, the liquid is drained towards the contact zones where the Laplace pressure is negative. A finite volume of liquid may condense on the grains as a result of surface roughness and high local capillary pressure, as observed in experiments [26]. We believe that in the annealing approach based on the minimisation of the total surface energy and with a net separation between liquid and vapour phases [26], the numerical roughness may lead to a similar effect whereas in LBM the fluid density can change continuously and it leads to a layer with density gradient at the interface.

The evolution of liquid clusters and internal stresses or pressures are monitored as a function of the amount of liquid $S$ using a floodfill algorithm. This classical digital image processing algorithm allows one to determine the area connected to each seed node. The following algorithm is a simple recursive implementation of the floodfill (without boundary conditions) in which we assume that pixel is an object embedding 3 variables $x, y$ and colour.
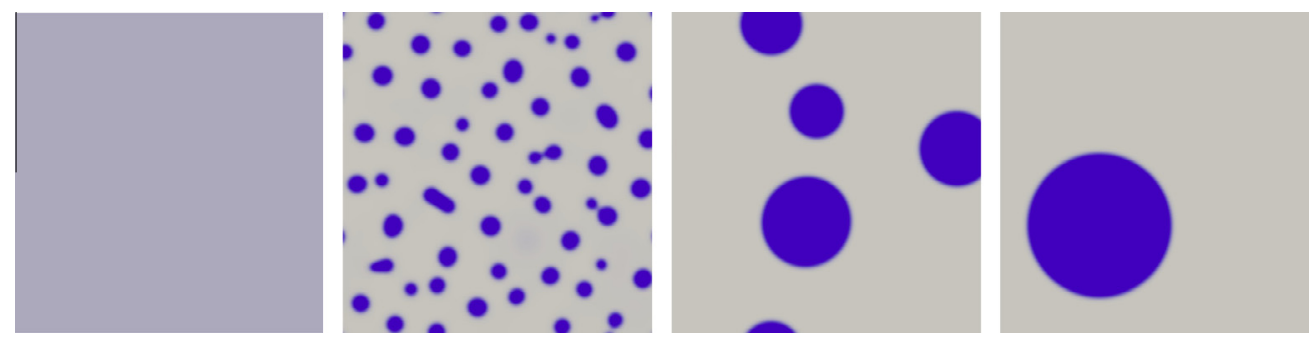

Fig. 3. From left to right: condensation and coalescence of liquid drops at constant volume. 
(a)

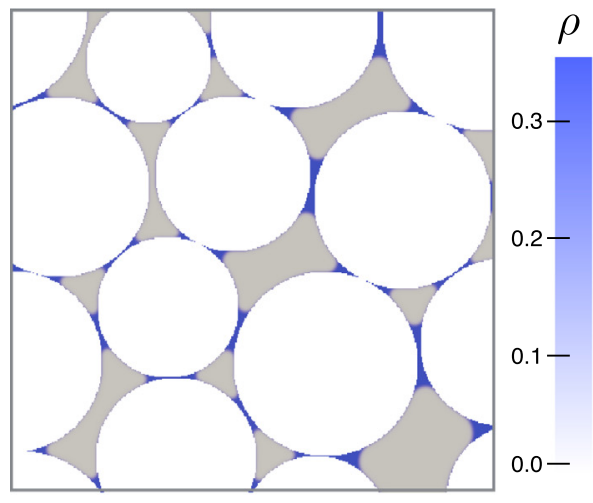

(b)

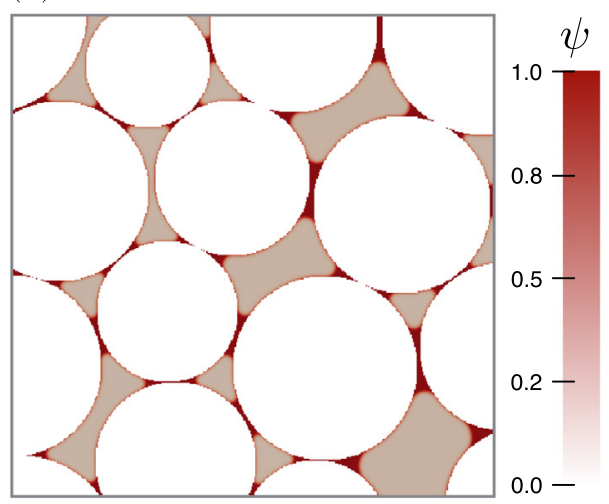

(c)

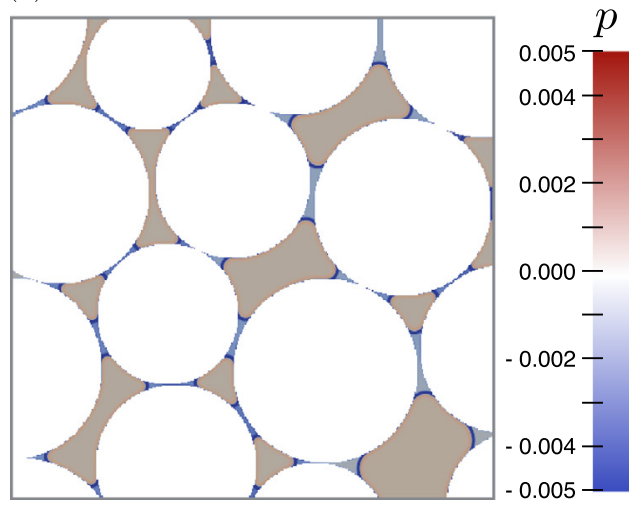

Fig. 5. Colours maps of a zoomed-in area for (a) the fluid density $\rho$, (b) the potential $\psi$ and fluid pressure $p$. (For interpretation of the references to colour in this figure legend, the reader is referred to the web version of this article.)

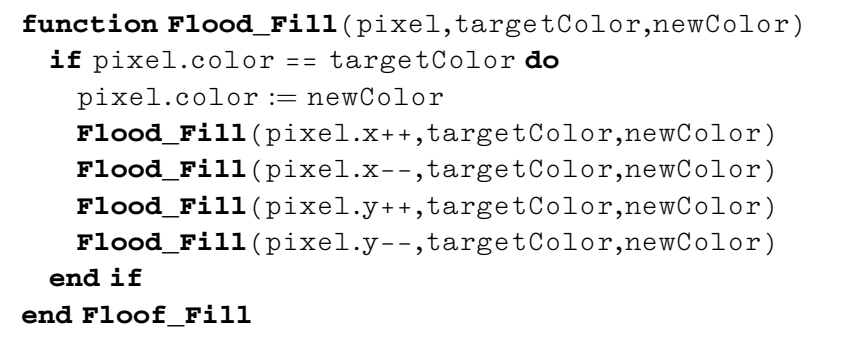

By using the contact points between grains as the initial seeds, we determine all independent clusters in the system for all values of $S$. The criterion used in the floodfill algorithm considers that two fluid pixels are connected if they are adjacent. This means that two liquid bridges belong to a single cluster if they are connected by at least a single liquid pixel. Using this procedure it is possible to determine the volume of the clusters or the connectivity of each cluster, i.e. the number of grains connected to a cluster; see Fig. 7.

In all simulations reported below, the grains are fixed, implying that the capillary forces resulting from liquid-gas surface tension acting at the triple points on the grains and Laplace pressure acting at the liquid-grain interface are fully balanced by the contact forces exerted by the neighbouring grains of each grain. The natural physical units of the system are the grain size $d$, liquid density $\rho_{\ell}$ and liquid-vapour surface tension $\gamma_{\ell v}$. Hence, in the following we normalise the lengths by $d$, the forces by $\gamma_{\ell v}$ and the stresses by $\gamma_{\ell v} / d$. Note that in 2D the surface tension is understood as "line tension" with the dimension of a force.

A single run of the program (parallelized with OpenMP) from dry to fully saturated pore space takes nearly one day on an Intel $2.5 \mathrm{GHz}$ Core i7. Animation videos of sample simulations can be found at the following address: http://www.cgp-gateway.org/ Video/Wet_Packings/.

\section{Liquid clustering}

Fig. 8 displays three snapshots of liquid clusters with increasing amount of liquid $S$. Snapshot (a) corresponds to the pendular state where the liquid is distributed in the form of binary bridges between grains. Snapshot (b) represents a typical example of the early stages of the funicular state where liquid clusters are connected to two or more grains whereas snapshot (c) represents the latest stages of the funicular regime where a large number of grains are immersed in the liquid phase. For saturation degree greater than in snapshot (c) the liquid clusters percolate throughout the packing and the mixture can be more appropriately described in terms of the distribution of grains and bubbles in a liquid.

The clustering state may be characterised by the proportion $C_{m}$ of liquid clusters connected to $m$ grains. In the same way, the grain environments can be described by the proportion $P_{n}$ of grains connected to $n$ independent liquid clusters. We will refer to $m$ as the "order" of a cluster. The average state of liquid connectivity is given by the average clustering order $\langle m\rangle=\sum_{m=2}^{m_{\max }} m C_{m}$ and the "wet" coordination number is $\langle n\rangle=\sum_{n=1}^{n_{\max }} n P_{n}$. Fig. 9 shows the evolution of $\langle m\rangle$ and $\langle n\rangle$ as a function of the amount of liquid $S$. In the pendular state, we have $\langle m\rangle=2$. As the amount of liquid increases, liquid clusters of increasing order are formed by the coalescence of clusters of lower order. As a result, $\langle m\rangle$ begins to increase slowly from 2 at $S \geqslant 0.15$ and diverges around $S=0.6$. As we shall see below, this point corresponds to the percolation transition of the liquid phase.

The wet coordination number $\langle n\rangle$ increases in the pendular state from zero to a maximum $(\simeq 3.5$ at $S \simeq 0.15)$ corresponding to the largest number of binary liquid bridges. Then, it declines down to $\langle n\rangle \simeq 1$ at $S \simeq 0.8$, which corresponds to a state where most grains are either immersed in liquid or in contact with the percolating liquid cluster and a binary bridge; see Fig. 8(d).

A percolation transition occurs in the liquid phase at $S \simeq 0.6$ as seen in Fig. 11 where the volume of the largest cluster $V_{\max }$ normalised by the total pore volume $V_{\text {pores }}$ is plotted as a function of $S$. Note that the process of the coalescence of primary liquid clusters begins well before with an increasing number of immersed grains. After transition, the liquid phase forms a single cluster and we have $V_{\max } / V_{\text {pores }} \simeq S$. The percolation transition reveals the highly nonlinear character of the pore-wetting process although the distribution is homogeneous in the bulk. This feature is a reflection of granular disorder. The fact that the transition 

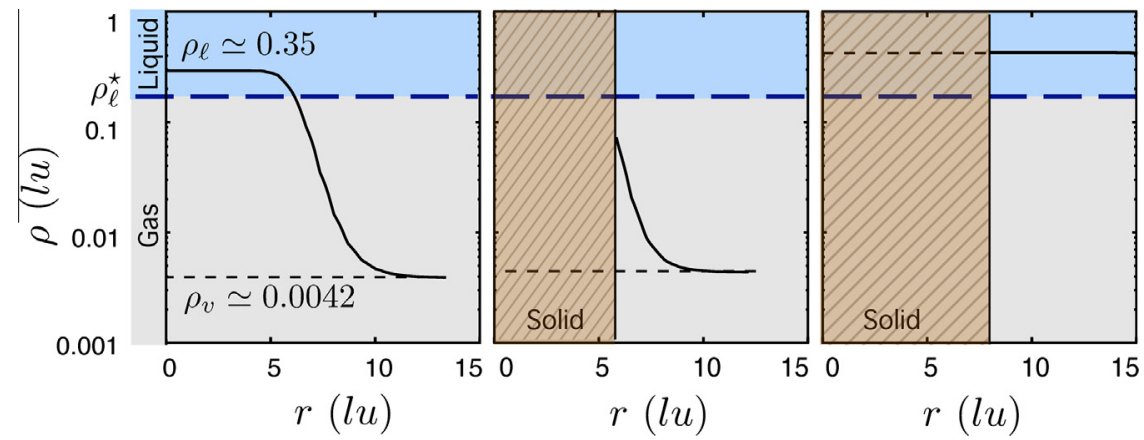

Fig. 6. Evolution of fluid density across liquid-vapour interface (a), solid-vapour interface (b) and solid-liquid interface (c) as a function of distance $r$ expressed in LBM units. $\rho_{\ell}^{\star}$ is the maximum spinodal density (see Fig. 2).

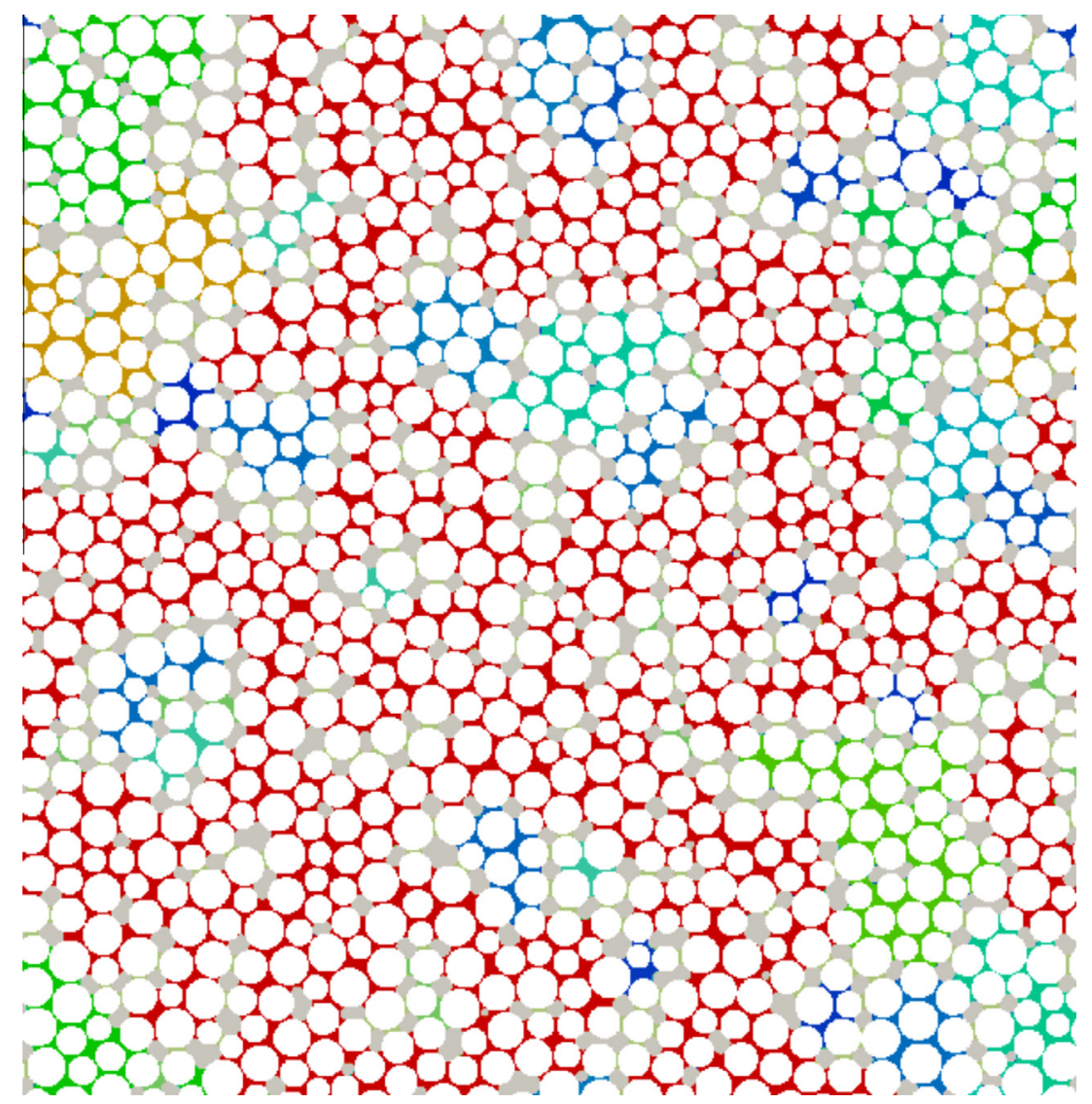

Fig. 7. Color map of the connectivity of liquid clusters at percolation. The colours are arbitrary and they design different clusters. (For interpretation of the references to colour in this figure legend, the reader is referred to the web version of this article.)
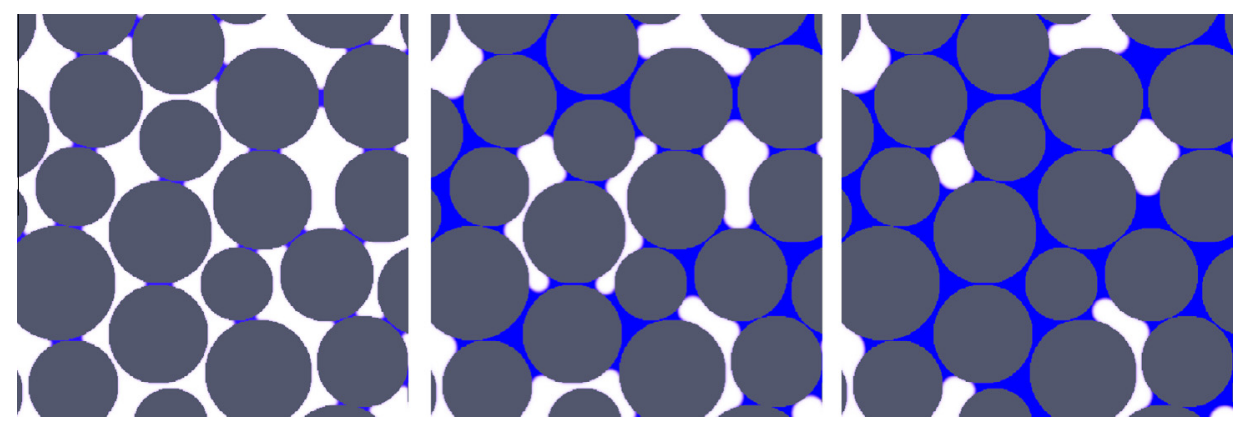

Fig. 8. Snapshots of liquid clusters with increasing amount of liquid. From left to right, $S=0.1, S=0.3$ and $S=0.7$. 


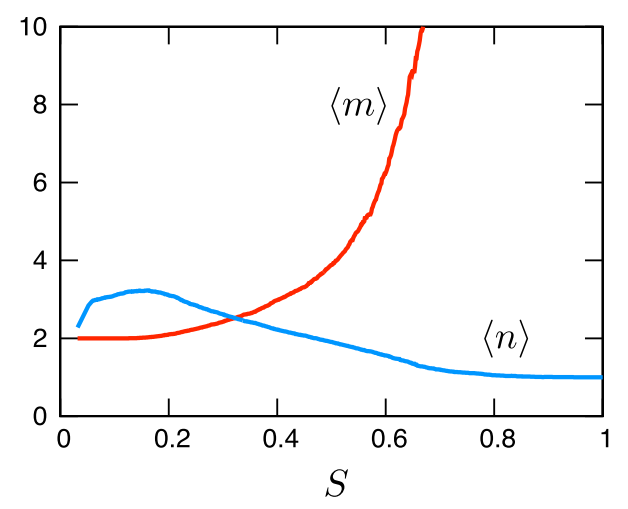

Fig. 9. Evolution of the average clustering order $\langle m\rangle$ and wet coordination number $\langle n\rangle$ as a function of the amount of liquid.

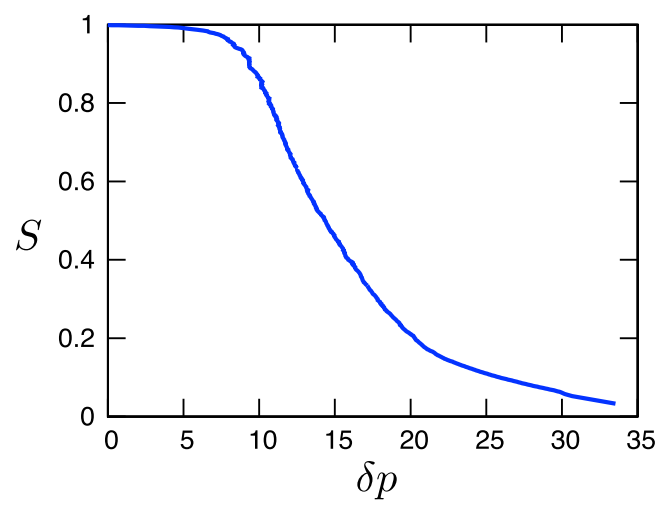

Fig. 10. The amount of liquid $S$ versus Laplace pressure difference $\delta p=p_{v}-p_{\ell}$, where $p_{v}$ is the mean vapour pressure and $p_{\ell}$ is the mean liquid (negative) pressure. The pressures are normalised by $\gamma_{\ell v} / d$.

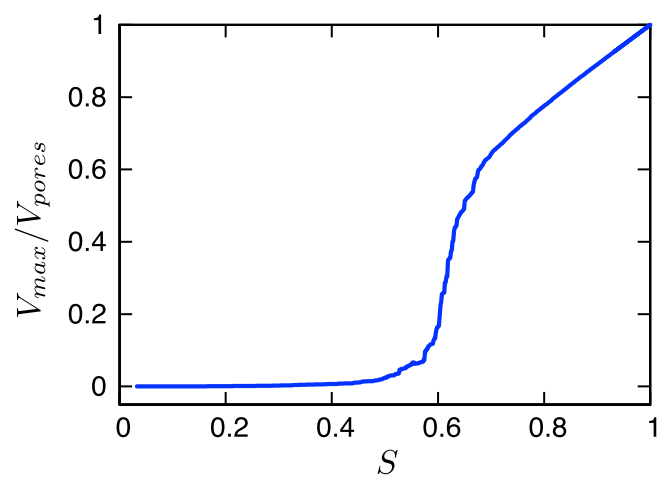

Fig. 11. Evolution of the normalised volume $V_{\max } / V_{\text {pores }}$ of the largest liquid cluster as a function of the amount of liquid $S$.

occurs far below full saturation $(S=1)$ means that the liquid can exist as a continuous phase without filling the pores.

The macroscopic states of water in soils are generally classified by considering the relationship between water content $S$, and the Laplace pressure difference (or suction) $\delta p=p_{v}-p_{\ell}$, where $p_{v}$ is the mean vapour pressure and $p_{\ell}$ is the mean liquid (negative) pressure. This retention curve is used to characterise different types of soil (depending on the grain size and shape distributions), but it has a generic form that is quite well reproduced by our simulations, as shown in Fig. 10. The two ranges $S<0.15$ and $S>0.9$ can be distinguished from the intermediate regime where $S$ increases rapidly as $\delta p$ declines with practically no signature of

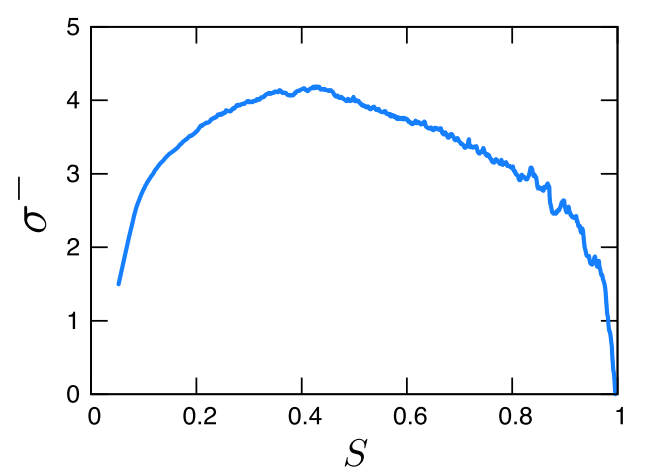

Fig. 12. Evolution of cohesive strength $\sigma^{-}$as a function of liquid content $S$.

the percolation transition evidenced for the largest liquid cluster in Fig. 11.

The largest values of $\delta p$ occur at the very early stages of the pendular state with small values of the radius of curvature of capillary bridges. In our simulations, as a result of the permeable layer introduced at the contact points allowing for fluid transfer between pores in $2 \mathrm{D}$, the largest value (in absolute value) of $\delta p$ reflects the minimal gap between grains. Up to $S \simeq 0.15$, the Laplace pressure declines almost linearly as $S$ increases as a result of the increase of the radius curvature of the capillary bridges.

\section{Cohesive strength}

The cohesive strength $\sigma^{-}$is the mean tensile stress due to the capillary action of liquid clusters on the grains. It was calculated from the forces exerted by the liquid on each grain and using the micromechanical definition of the stress tensor [27]. In this calculation, only the cohesive component of the stress tensor, resulting from the tensile action of the capillary forces on the grains, are taken into account. Conversely, all liquid clusters are in equilibrium on the action of the reaction forces exerted by the immobile grains. Fig. 12 shows that the cohesive strength (in absolute value) increases beyond the pendular state, though at a lower rate, up to a maximum (slightly above the strength at the end of the pendular state) at $S \simeq 0.4$ before falling off again as result of the pressure drop inside the liquid clusters. We find that the peak value of $\sigma^{-}$ and the saturation 0.4 at which it occurs have no signature on the evolution of the microstructure but they rather result from a competition between the monotonic decrease of liquid pressure and increase of grain-liquid interface [8]. This process coincides with an increasing number of immersed grains and a decreasing number of triple points. In this way, the peak cohesion divides the funicular regime into two different regimes with ascending cohesion in the early funicular regime and descending cohesion in the late funicular regime.

\section{Conclusions}

In this paper, a brief description of the multi-component Lattice Boltzmann Method was presented in view of application to unsaturated granular materials. LBM simulations of homogeneous capillary condensation were used to create homogeneous distributions of liquid in the form of capillary bridges and clusters. We analysed the clustering of liquid and the evolution of Laplace pressure as a function of the amount of liquid in a 2D granular packing. The coalescence of binary bridges begins for an amount of liquid $S \simeq 0.15$ where the mean order $\langle m\rangle$ of liquid clusters begins to increase whereas the volume of the largest cluster remains nearly constant up to $S \simeq 0.6$. This shows that the funicular state in this range may simply be seen as a juxtaposition of hardly touching binary bridges, 
in agreement with the experiments of Scheel et al. [6]. The largest cluster begins to grow after this primary phase of coalescence and the liquid clusters percolate throughout the system. In this late funicular state, an increasing number of grains are immersed in the liquid and the vapour phase is in the form of bubbles connected to two or more grains.

The Laplace pressure declines during saturation as a function of $S$ with a shape very similar to measured retention curves in soil mechanics. The evolution of the connectivity of liquid clusters evidenced by simulations in this work for all degrees of saturation (from binary bridges to full saturation of pores) indicates that four states should be distinguished in the description of unsaturated soils: (1) pendular state, corresponding to binary liquid bridges, (2) primary funicular state, where the capillary bridges join one another without efficient filling the volume, (3) secondary funicular state, where the liquid volume percolates and many particles are fully immersed, (4) bubble state, characterised by bubbles connected to two or more grains.

The LBM/DEM approach presented in this paper, provides a powerful toolbox for the investigation of unsaturated soils [28]. The effects of void ratio, particle size distribution and particle shapes can be simulated and characterised in terms of both their retention curves and cohesive strength curves. We do not expect a fundamental difference with the picture described in this paper. But it is clear that liquid distribution in samples of highly polydisperse systems, for example, is much more inhomogeneous and may also lead to a different level of cohesion than in weakly polydisperse systems. Furthermore, this approach makes it possible to simulate the evolution of the liquid during drainage from a boundary or under the action of gravity. It would be interesting to compare the multi-component LBM in this case with the predictions of annealing approaches and experiments in this context.

\section{References}

[1] Iverson RM, Reid ME, Iverson NR, LaHusen RG, Logan M, Mann JE, et al. Acute sensitivity of landslide rates to initial soil porosity. Science 2000;290 (5491):513-6. http://dx.doi.org/10.1126/science.290.5491.513.

[2] Ghestem M, Sidle RC, Stokes A. The influence of plant root systems on subsurface flow: implications for slope stability. Bioscience 2011;61 (11):869-79. http://dx.doi.org/10.1525/bio.2011.61.11.6.

[3] Ruiz T, Rondet E, Delalonde M, Desfours JP. Hydro-textural and consistency surface states of humid granular media. Powder Technol 2011;208(2):409-16. http://dx.doi.org/10.1016/i.powtec.2010.08.036.

[4] Richefeu V, El Youssoufi MS, Radjai F. Shear strength properties of wet granular materials. Phys Rev E 2006;73(5):051304. http://dx.doi.org/10.1103/PhysRevE $\underline{73.051304 .}$.
[5] Mitarai N, Nori F. Wet granular materials. Adv Phys 2006;55(1-2):1-45. http:/ dx.doi.org/10.1080/00018730600626065.

[6] Scheel M, Seemann R, Brinkmann M, Di Michiel M, Sheppard A, Breidenbach B, et al. Morphological clues to wet granular pile stability. Nat Mater 2008;7 (3):189-93. http://dx.doi.org/10.1038/nmat2117.

[7] Mitchell J, Soga K. Fundamentals of soil behavior. New-York, NY: Wiley; 2005.

[8] Delenne JY, Richefeu V, Radjai F. Liquid clustering and capillary pressure in granular media. J Fluid Mech 2015;762(5).

[9] Cundall P, Strack O. A discrete numerical model for granular assemblies. Geotechnique 1979;29:47-65.

[10] Radjai F, Dubois F, editors. Discrete-element modeling of granular materials. Wiley; 2011.

[11] Guyon E, Hulin J-P, Petit L, Mitescu CD. Physical hydrodynamics. Oxford University Press; 2001.

[12] He X, Doolen GD. Thermodynamic foundations of kinetic theory and lattice Boltzmann models for multiphase flows. J Stat Phys 2002;107:309-28. http:/ dx.doi.org/10.1023/A:1014527108336.

[13] Succi S. The Lattice Boltzmann equation for fluid dynamics and beyond. Oxford University Press; 2001.

[14] Bhatnagar PL, Gross EP, Krook M. A model for collision processes in gases. I. Small amplitude processes in charged and neutral one-component systems. Phys Rev 1954;94(3):511-25.

[15] Satoh A. Introduction to practice of molecular simulation. USA: Elsevier; 2011.

[16] He X, Chen S, Zhang R. A lattice Boltzmann scheme for incompressible multiphase flow and its application in simulation of Rayleigh-Taylor instability. J Comput Phys 1999;152(2):642-63.

[17] Sukop M, Or D. Lattice Boltzmann method for modeling liquid-vapor interface configurations in porous media. Water Resour Res 2004;40:W01509.

[18] Huang H, Lu X. Relative permeabilities and coupling effects in steady-state gas-liquid flow in porous media: a lattice Boltzmann study. Phys Fluids 2009;21(9):092104.

[19] Shan X. Lattice Boltzmann model for simulating flows with multiple phases and components. Phys Rev E 1993;47(3):1815-9. http://dx.doi.org/10.1103/ PhysRevE.47.1815.

[20] Shan X. Simulation of nonideal gases and liquid-gas phase transitions by the lattice Boltzmann equation. Phys Rev E 1994;49(4):2941-8. http://dx.doi.org/ 10.1103/PhysRevE.49.2941.

[21] Shan X, Doolen G. Multicomponent lattice-Boltzmann model with interparticle interaction. J Stat Phys 1995;81:379-93. http://dx.doi.org/10.1007/ BF02179985.

[22] Swift M. Lattice Boltzmann simulations of liquid-gas and binary fluid systems. Phys Rev E 1996;54(5):5041-52. http://dx.doi.org/10.1103/PhysRevE.54.5041.

[23] Kupershtokh A, Medvedev D, Karpov D. On equations of state in a lattice Boltzmann method. Comput Math Appl 2009;58(5):965-74.

[24] Yuan P, Schaefer L. Equations of state in a lattice Boltzmann model. Phys Fluids 2006;18:042101.

[25] Huang H, Li Z, Liu S, Lu X. Shan-and-Chen-type multiphase lattice boltzmann study of viscous coupling effects for two-phase flow in porous media. Int J Numer Meth Fluids 2008;61(3):341-54.

[26] Han M, Youssef S, Rosenberg E, Fleury M, Levitz P. Deviation from Archies law in partially saturated porous media: wetting film versus disconnectedness of the conducting phase. Phys Rev E 2009;79:031127.

[27] Staron L, Radjai F, Vilotte J-P. Multi-scale analysis of the stress state in a granular slope in transition to failure. Eur Phys J E Soft Matter 2005;18 (3):311-20. http://dx.doi.org/10.1140/epje/e2005-00031-0.

[28] Roux J-N. IlA numerical toolkit to understand the mechanics of partially saturated granular materials, focus on fluids. J Fluid Mech 2015;770:1-4. http://dx.doi.org/10.1017/ifm.2015.66. 